\title{
DEUDAS E INDEMNIZACIONES \\ ASPECTOS NEGATIVOS DE LA HERENCIA DE LOS PRIMEROS CONDES DE UREÑA
}

\author{
ANA Viña BRTTO \\ Universidad de La Laguna \\ (Tenerife, Canarias)
}

Los fondos notariales y muy especialmente los testamentos constituyen una fuente interesante para conocer las actitudes y comportamientos individuales o colectivos de la sociedad de una época. En los años finales de la Edad Media e inicios de los tiempos modernos los testamentos se concebían no exclusivamente como un acto de derecho privado, sino como un documento en el que se reflejaba una finalidad de tipo religioso que resulta evidente a través del análisis de sus diferentes cláusulas, pues no podemos olvidar que la redacción del testamento constituia un paso imprescindible para una buena muerte en la que se preparaba el terreno para después del óbito, de ahí que junto a las misas por su alma se recuerde a los padres y parientes a quienes también se les ofrecen misas, intentando paliar las deficiencias que quedaron' pendientes en vida. Ello explica que el moribundo "no es un actor sino un mero testigo en el combate entre dos sociedades, la de los demonios y la de los ángeles".

Estos aspectos de tipo religioso junto a los asuntos terrenales: reconocimiento de deudas, nombramiento de herederos, distribución del

'Maria Josefa PAREJo DELGADO, Costumbres mortuorias recogidas en los testamentos Ubetenses de fines de la Edad Media, "Actas del VI Coloquio Intemacional de Historia Medieval Andaluza", Málaga, 1991, pp. 319 y 88.

-Anuario de Estudios Madievales": 25 (1995) 
patrimonio, etc., que tenfan como finalidad el regular las sucesiones y prevenir disputas familiares, constituyen los dos elementos básicos de las disposiciones testamentarias y ambos recaerán sobre los herederos del difunto.

Un análisis de los testamentos de la nobleza bajomedieval castellana nos permite observar claramente este fenómeno, siendo cuantiosas las deudas a las que tienen que hacer frente los herederos, asl como una serie de compensaciones que estos últimos se ven obligados a realizar ya sea a particulares o a instituciones, a fin de atenuar los efectos de penitencias mal cumplidas.

Uno de los casos en que se observa claramente este fenomeno es en el testamento de don Pedro Girón, Maestre de Calatrava ${ }^{3}$. Obviaremos en este análisis la finalidad estrictamente religiosa del testamento asI como el reparto de la herencia entre sus hijos a fin de perpetuar su linaje y centraremos nuestro estudio en las compensaciones que los herederos del Maestre, y muy especialmente su segundo hijo, don Juan Téllez Giron, tuvo que realizar a fin no sólo de dar cumplimiento a las disposiciones testamentarias de su progenitor, sino sobre todo para que su padre, ya fallecido, alcanzase el perdón de algunas personas o colectivos que durante su vida el Maestre no pudo obtener. Estas deudas y compensaciones se tradujeron en una serie de gastos que el segundo conde de Ureña hubo de asumir a costa de su propio patrimonio personal.

Tanto las deudas como las compensaciones económicas de las que se hizo cargo don Juan Téllez-Giron, tras el fallecimiento de su padre, podemos situarlas en tres niveles. A nivel particular que ejemplificaremos en las compensaciones a Juana González; a nivel institucional simbolizada en la fundación del pósito de Jaén y en tercer lugar encontramos la ejecución material de una de las disposiciones testamentarias de su padre, la fundación de una capellania en Belmonte.

Las referencias a deudas e indemnizaciones son frecuentes no sólo en este caso sino en general en toda la nobleza bajomedieval, asi observamos como era práctica habitual que los testamentarios, en este caso de don Pedro

\footnotetext{
${ }^{2}$ Empleamos la terminología de A. ALEMAN ILUN, La muerte en la sociedad murciana a fines del Ansiguo Régimen, "Contrastes. Revista de Historia Modems", 3-4 (1987-1988), pp. 71.90.

"Ana VINA BRTTo, El testamento de don Pedro Girón, Maestre de Calatrava, "Anuario de Estudios Medievales", 19 (1989), pp. 493 y ss.
} 
Girón, hicieran frente a importantes cantidades de dinero a fin de indemnizar los daños causados en acciones de guerra, como ocurrió por ejemplo en el año 1471 cuando el Chanciller mayor de Calatrava libró a frey Bartolomé Almodóvar, sacristán del convento de Calatrava, unos 33.000 mrs. para reparar los daños causados por el Maestre en los campos de Calatrava y Ciudad Real en su anterior enfrentamiento con el Clavero por la posesión del Maestrazgo4.

Como consecuencia directa de la agitada vida política del Maestre de Calatrava, que participo activamente en numerosos saqueos, rebeliones, guerras, etc., el segundo conde de Ureña se vio obligado a efectuar una serie de remuneraciones de tipo económico a particulares e instituciones. Estas compensaciones eran de obligado cumplimiento a fin de contribuir a la salvación del alma de su padre, lo que nos lleva a afirmar que la herencia que los condes de Ureña recibieron presenta una serie de aspectos negativos que es necesario tener en cuenta.

Si bien es verdad que don Juan Téllez-Girón fue eximido, por indulgencia del papa Inocencio $\mathrm{VII}^{\mathrm{s}}$, de la obligación de compensar los daños ocasionados por su padre, que fue perdonado de sus correrías por Toledo, Jaén y Ciudad Real, cuando la citada indulgencia se produce, en el año 1490, ya el segundo conde habra abonado importantes cantidades de dinero a particulares para hacer frente a los daños ocasionados en Toledo y Ciudad Real, pero no en Jaén a quién resarcirá espléndidamente varios años después.

El primer caso que analizamos son las compensaciones a particulares que hemos centrado en las indemnizaciones a Juana González. Constituye éste un caso significativo ya que la pérdida de sus bienes en favor de don Pedro Girón se produjo en el año 1459 y no será hasta año 1470 cuando se inicie el litigio con los herederos del Maestre. Los citados bienes les fueron retornados varios años más tarde.

El desarrollo de este interesante litigio es semejante a los acaecidos en esta etapa, la solicitud en la villa de Almagro el 25 de julio del año 1470, por parte de la demandante, la citada Juana González mujer del bachiller Alvaro González, ya fallecido; la presentación de testigos ante el alcalde de la villa y el escribano público, Juan de Perea y Gonzalo Gómez de Carrión,

\footnotetext{
"Archivo Histórico Nacional (AHN), Osuna, legajo 2, n. 14. y n. 13 a-c.

51490, s.m., s.d. Roma. Biblioteca Nacional, Ms. 6776, fol. 96.
} 
respectivamente. A continuación se procedía a la toma de juramentos cuyo testigo principal era el cura de la villa, Juan Ruiz. La parte demandada estaba representada por el antiguo recaudador del Maestre, Gonzalo de Párraga, que continuaba en esos momentos al servicio de la casa de los condes de Ureña.

Tras esta primera fase, con posterioridad concretamente el 1 de marzo del año 1472, compareció de nuevo Juana González, viuda del bachiller Alvaro González Basbudillo, con dos nuevos testigos vecinos de Almagro, Alvaro Núñez del Castillo y Juan Grande. Sin duda alguna esta segunda comparecencia de la que tenemos referencia marcó la solucion definitiva del conflicto.

A través de su análisis que sigue las fórmulas habituales "fizieron juramento en forma... jurado e preguntado por el juramento que fizo dixo preguntado si sabe...", vemos claramente no sólo los bienes usurpados, sino las razones que los motivaron y sobre todo y más importante aún que el conde de Ureña tuvo que hacerse cargo de las indemnizaciones derivadas de la actuación de su progenitor, con la finalidad como ya hemos señalado de contribuir a limpiar la mala imagen que su padre dejo en vida.

En cuanto a los testigos presentados por la parte demandante en esta actuación de 1472, el primero de ellos, Alvaro Núñez del Castillo, confirm6 que en el año de 1459 el bachiller Fernando Draz de Madrid, alcalde mayor, le llamo para que fuese como testigo a la posada donde residfa el Bachiller Alvaro González Basbudillo; en la citada posada hicieron un inventario de todos sus bienes (Anexo 1) de los que luego se apropiaron los criados del Maestre, Fernando de las Espuelas y Pedro de Tena. Este testigo manifesto desconocer los motivos que llevaron al Maestre de Calatrava a tomar las pertenencias del bachiller González Basbudillo, aunque apunta que pudieran haber sido ciertas palabras que el Bachiller tuvo con el sacristán del convento, quien lo comunic $\sigma$ al Maestre, según se deduce de un testimonio que presentó Gonzalo de Carrión que a su vez era el escribano público.

La declaración del otro testigu, Juan Grande, es semejante a la anterior y si bien señaló que desconocía el valor total de los bienes usurpados si manifestó que Juana González tenf́a su casa "bien arreada de muchas cosas y arreos tanto e quanto la mas e mejor de los vezinos de Almagro".

A través de la descripción de los bienes muebles y raf́ces, que según la demanda presentada usurpo don Pedro Girón en sus correrfas por Almagro, podemos conocer la dotación de una de las mejores casas de la 
ciudad en la segunda mitad del XV. No era frecuente en la época ni la cantidad ni la calidad de los bienes que figuran en el inventario aludido, tanto en lo referente a la denominada ropa de casa como en los útiles de la misma: cuchillos, sillas, copas de plata, platos vidriados, etc.. como tampoco lo eran las provisiones que guardaba: 600 fanegas de trigo, 50 fanegas de cebada, 6 tinajas llenas de vino, aparte de los animales, sin olvidar las arras, alhajas, o la biblioteca que en nada tenfa que envidiar a las que posera la alta nobleza en la misma época: ocho volúmenes de libros grandes o pequeños, siete partidas en papel, Enriques del primero al cuarto, Digesto, Código, etc. en papel, asf como libros de lectura en pergamino, dos decretales, cuadernos de vidas de santos,....

Desconocemos la cuantía economica total en que fue tasada esta apropiación indebida, pero suponemos que debio ser elevada y sobre todo que la misma repercutió en las arcas de los condes de Ureña, pues son ellos los que tuvieron que hacer frente a las deudas o mejor indemnizaciones contrardas por su padre que fueron numerosas ${ }^{6}$.

Un segundo caso interesante y que por supuesto no fue el único, hace también referencia a los gastos efectuados por el segundo conde de Ureña para resarcir los daños ocasionados por su padre, aunque en este caso los beneficiarios no son individuos aislados sino la totalidad de los vecinos de una ciudad, la de Jaén.

Las razones que motivaron los agravios de don Pedro Girón a la ciudad de Jaén son bien conocidos ${ }^{7}$. En síntesis podríamos decir que en el año 1465 don Pedro Giron lideraba el bando favorable al infante don Alfonso frente a los partidarios del monarca Enrique IV. Este enfrentamiento entre bandos contrarios alcanzo su punto álgido en tierras del obispado de Jaén donde el Maestre de Calatrava causo "bolliçios, escandalos... facen muchos ayuntamientos e robos e fuerças", pues como señala la crónica del Condestable "... e el dicho Maestre de Calatrava vino a su tierra que es al obispado de Jaen e allego fasta setecientos u ochocientos rocines"

\footnotetext{
'Otro ejemplo es la reclamación de Catalina de Guzmán que reclamó por sus cosas y las de su padre, usurpadas por don Pedro Girón, y que les fueron devueltas tras dar fe de esta fechoría dos criados del Maestre. Cit. Francisco de UhAGON, Ordenes Militares, Madrid, 1989, p. 98.

'Ara ViÑ Brito, Morón y Osuna en la Baja Edad Media, Sevilla, 1991. José RodRÍGUez MouNA, El reino de Jaén en la Baja Edad Media, Graneda, 1978, p. 146.

'P. Escavias, Hechos del Condestable don Miguel Lucas de Iranzo, Madrid, 1940, Cap. XXIII, Pp. 253.
} 
La actividad desplegada por don Pedro Girón en Andalucla como capitán del bando favorable al príncipe don Alfonso y los numerosos enfrentamientos con el bando contrario, proporcionaron a la ciudad de Jaén, a largo plazo, una serie de compensaciones por parte de los herederos del Maestre, los cuales, casi 30 años después de producidas las correrfas de don Pedro Girón, indemnizaron a la ciudad con la construcción de un posito en desagravio de los daños causados a la localidad y a sus vecinos por parte del Maestre?.

El sistema de pósitos o cuotas obligatorias de almacenamiento controlado por los municipios para prever la escasez de trigo, comenzo a desarrollarse en la Corona de Castilla ${ }^{10}$ entre los años 1474 y 1503 . En este caso concreto, si bien la finalidad del posito era mantener el acopio de trigo para repartirlo a los labradores en condiciones módicas durante los períodos de carestra, tanto la construcción como el control del pósito de Jaén estuvo a cargo del segundo conde de Ureña, pues como ya hemos manifestado el motivo de su edificación fue el de resarcir los daños ocasionados por su padre a esta ciudad.

Si bien puede llamarnos la atención la demora en la compensación, de 1465 a 1494, lo es más porque al menos desde hacía cuatro años el segundo conde de Ureña habla sido liberado, por indulgencia de Inocencio VII, de su obligación de resarcir los daños ocasionados por su padre; a pesar de ello, don Juan Téllez-Girón cumplió con el compromiso adquirido tal como se deduce de la promesa que hizo a la ciudad de Jaén de $260.000 \mathrm{mrs}$. para la compra de pan y la construcción de un granero". La citada fundación, tal como figura en un traslado posterior de 1553, contaba con la preceptiva "comisión real de sus magestades sellada con su real sello y librada de los señores del su consejo" 12 .

Si analizamos detalladamente las vicisitudes que corrio este posito, cabe señalar en primer lugar que en la misma fecha de la promesa de edificación del posito por don Juan Téllez Girón, se emitió una provisión y

\footnotetext{
'Don Juan Téllez-Girón mandó edificar este pósito en marzo del año 1494.

${ }^{10}$ Según señala Miguel Angel LADERO QUESADA, La Hacienda real de Castilla en el siglo $X V$, Tenerife, 1973, p. 224.

"Anchivo General de Simanca, RGS, 3-1494-XI. Cit. Jose RodRIGUEZ MOUNA, Bandos de las ciudades en el also Guadalquivir, Siglos XV y XVI. Repercusiones, "Actas del VI Coloquio", ob. cit. pp. 537 y 28.

${ }^{12} \mathrm{AHN}$, Oxuna, leg. 35, n. 48, fol. 93 y so.
} 
cédula de los RR.CC. (Medina del Campo, 7-3-1494) en la que se obligó al conde a sustentar y dar aumento al citado pósito.

Un año antes, en 1493, los vecinos de cada una de las collaciones de la ciudad de Jaén determinaron realizar una carta de perdón al Maestre de Calatrava, concretamente las de San Bartolomé, la Magdalena, San Andrés, San Alfonso, San Miguel, San Llorente, Santiago, San Juan, Santa María, San Pedro y la de Santa Cruz ${ }^{13}$. Las citadas cartas de perdón se llevaron a cabo a cambio de los $200.000 \mathrm{mrs}$. que el señor conde de Ureña mandó dar para trigo, además de otros $60.000 \mathrm{mrs}$. para comprar una casa en que guardarlo "e que se faga un deposito de pan como se faziere y este presente para siempre por la necesidad..." 14 .

Junto a estas cartas de perdón por collaciones figuran otras concedidas por particulares, como la de Ana Sánchez, vecina de Jaén, y sus hijos por $11.000 \mathrm{mrs}$. que le fueron resarcidos por el segundo conde de Ureña.

Una vez donado el numerario suficiente se procedió a la creación del pósito para almacenar el trigo que se comprase fuera de la localidad y sobre todo, para que el trigo almacenado se destinase al abastecimiento de la población que de otra manera no hubiese podido adquirirlo. Desconocemos el precio exacto de la fanega de trigo en este año de 1494, pero suponemos que la donación del conde de Ureña fue importante si la comparamos con la que pocos años después otorgó Beatriz Pacheco a Carmona, $175.000 \mathrm{mrs}$. con los que se compraron 3.000 fanegas de trigo en Sevilla ${ }^{15}$. La compra

\footnotetext{
${ }^{13}$ Las citadas cartas de perdón de las collaciones fueron hechas ante los escribanos correspondientes:

San Bartolomé ante Francisco de Soleda.

La Magdalena ante Matco de Espinosa.

San Andrés ante Ruy Gomes.

San Alfonso ante Femán Gomes de Molina.

San Miguel ante Perp Ximenes.

San Llorente ante Blas Gomes.

Santiago ante Juan de Castro.

San Juan ante Andrés de Aranda.

Santa Maria ante Femán Gomes de Molina.

San Pedro ante Pero Nuñes de Soria y

Santa Cruz ante Gonzalo Ruis.

${ }^{14} \mathrm{AHN}$, Osuna, leg. 35, n. 48.

${ }^{15}$ Manuel GONZÁlez Jiménez, El Concejo de Carmona a fines de la Edad Media (14641523), Sevilla, 1973, p. 263.
} 
del pan en Jaén debráa realizarse a primeros de agusto según indicación expresa de don Juan Téllez-Girón.

Los monarcas castellanos ordenaron, una vez erigido el pósito, yue este se tuviera en pie y sobre todo que el juez de términos, el licenciado Cuéllar, "tome las quentas del posito e gastos della".

Las referencias en la documentación a la actuación del citado juez son abundantes, estando asistido en su labor por el conde de Ureña el cual envió como delegado suyo al licenciado Juan Caro de Sotomayor. Según la normativa que regfa el funcionamiento del pósito, correspondlia a don Juan Téllez Girón como fundador del mismo "el enviar a un criado de su señorfa", ya que al conde "le pertenece el estar y asistir y nombrar persona que por el asista a las quentas y alcançes del posito...", mientras que el regimiento nombraba, por su parte, a un mayordomo o a otra persona cuya función era la de encargarse del pan y de los maravedfs. A pesar de este nombramiento efectuado por el regimiento, da la impresión que la actividad desarrollada por el delegado municipal estaba bastante coartada, pues tal como podemos inferir de traslados posteriores, era don Juan Téllez-Girón quien nombraba a la persona encargada de controlar la cantidad de pan y maravedís que tenía el pósito, asi como de vigilar estrechamente el reparto de trigo y sobre todo controlar a aquellas personas "que le deven algun trigo y mrs.".

Sin duda alguna, el delegado del conde era la autoridad principal puesto que "los señores del ayuntamiento desta ciudad (Jaén) avrogaron en favor de los señores del estado de Ureña", lo que significa un control efectivo por parte del segundo conde de Ureña, al margen de cualquier injerencia del regimiento. Esta situación puede explicar, en parte, que a lo largo del siglo XVI sean frecuentes los requerimientos a los encargados del pósito para que cobren las deudas pendientes a fin de invertir el dinero recaudado en la compra de pan, estipulándose que la mejor fecha para hacerlo era en agosto por ser el mes de la cosecha lo que supone que el trigo se vendería a unos precios más moderados.

La preocupación tanto del segundo conde de Ureña como posteriormente de sus sucesores por el buen funcionamiento del pósito fue una constante, como lo demuestra el hecho de que a finales de la centuria el duque de Osuna visitó el pósito de Jaén y se justificó liquidar las cuentas que tenfa y que eran de 26532 fanegas y algunos celemines de trigo, ascendiendo su valor en metálico a $9.653 .640 \mathrm{mrs}$. 
En este caso de Jaén, la fundación del pósito funcionaba más como una obra de beneficencia, al menos en los primeros momentos, que como un posito municipal, pero lo que si es cierto es que mereció toda la atención de los condes de Ureña (Anexo 2) y por supuesto la intervención real, obligando en ocasiones a "los propios y rentas de Jaen e hazer escritura de obligacion al que mi señor vaya (se refiere al conde de Ureña) por ser razon de dicho posito". En la segunda mitad del XVI podemos afirmar que los monarcas efectuaron un seguimiento más detallado de la evolución del pósito como consecuencia, posiblemente, de las frecuentes quejas que presentaron los vecinos de Jaén ante la mala administración que de él se hacia, y sobre todo porque los regidores, jurados y otros caballeros se reparten entre sf los mrs. y el pan del pósito. Sirva como ejemplo la petición de Fernando de Paredes ante Carlos I, quien obligo a devolver lo usurpado ${ }^{16}$ y que los mrs. se depositaran en el "cofre que para ello esta consebido".

El tercer caso que analizamos, al igual que los dos expuestos anteriormentc, forman parte de las numerosas deudas e indemnizaciones que los herederos del Maestre tuvieron que hacer frente ya sea como compensacion por dafios de guerra o, como en este caso concreto, para cumplir una disposición testamentaria que no le reportó ningún beneficio a la futura casa de Osuna, sino todo lo contrario una disminución de sus rentas patrimoniales.

Nos estamos refiriendo al cumplimiento, por parte del segundo conde de Ureña, de la fundación de una capellanía en Belmonte en memoria de sus abuelos.

Es habitual encontrar en los testamentos de la nobleza castellana bajomedieval que las personas que estaban proximas a morir donaran importantes cantidades de dinero para la iglesia en la que su cuerpo iba a ser enterrado, que en el caso concreto de don Pedro Girón era el convento de su propia Orden, la de Calatrava. Junto a esta disposición que afectaba a su propio enterramiento, encontramos el recuerdo hacia sus padres en cuya memoria el Maestre orden 6 que a su muerte se hiciese una capellanfa en Belmonte, dotada con 6.000 mrs. de renta de heredades ${ }^{17}$.

\footnotetext{
${ }^{16}$ Valladolid, 7-12-1553 (AHN, Osuna, leg. 35). 500.

"Ana VIÑA BRTo, El Testamenta de don Pedro Girón, Maestre de Calatrava, ob. cit., p.
} 
La citada fundación prescrita en el año 1466, no se hizo efectiva hasta el año 1502. Las razones que motivaron esta tardanza vienen determinadas en primer lugar por la minoria de edad y temprana muerte del primer conde de Ureña, a lo que se añade el hecho de que en los primeros años de don Juan Téllez-Girón al frente del señorfo, tuvo que dedicar todos sus esfuerzos a mantener la integridad del mismo, a la reconciliación con los Reyes Católicos y a subsanar los agravios cometidos por su padre que le fueron reclamados, como ya hemos señalado en los dos casos precedentes.

El segundo conde de Ureña ejecutó literalmente esta cláusula testamentaria de don Pedro Girón. según la cual sus herederos "vieren que la dicha capellania sea meior servida". y para ello la doto con $12.000 \mathrm{mrs}$. cada año "en la renta maior de la veyntena y peso de la villa de Osuna" (Anexo 4). hasta que pudiese dotarse la citada capellanfa en la villa de Belmonte. lo que posiblemente no ocurrió puesto que la citada villa no era de su propiedad y además si hien la capellanfa hasta esos momentos, año 1502, parece que estaha sin dotación. unos años antes su tío Juan Pacheco habra dejado un juro de $25.000 \mathrm{mrs}$. cuyos heneficiarios eran los clérigos de la iglesia de San Bartolome de Belmonte, a fin de rezar por su alma y la de sus padres y abuelos ${ }^{18}$.

Para que esta donación fuera efectiva don Juan Téllez-Girón, además de la carta de concesión al prior y cabildo de Belmonte de los $12.000 \mathrm{mrs}$., dirigio otra a los arrendadores de la renta mayor de Osuna para hacer cumplir la citada concesión, especificando en la misma que "no lleve ni descontento dellos ni ningunos derechos" ${ }^{\prime 9}$.

Si bien es verdad que un análisis exhaustivo del testamento de don Pedro Giron nos ha llevado a atirmar que el principal apartado es el reparto de la herencia entre sus hijos ${ }^{20}$, junto a la salvación de su alma y mandas pras, instituyendo el mayorazgo para su hijo primogénito y determinando así la creación de su propio linaje familiar, no podemos olvidar que al igual que sucedia con otros linajes nobiliarios, los herederos son responsables de las deudas contrafdas por sus progenitores y también era su obligación el hacer frente a cuantiosas indemnizaciones para resarcir actuaciones poco ortodo-

\footnotetext{
19 Alfonso Fraico Silva, Los testainentos de Juan Pacheco (1470-1472), "Congreso de Historia del Señorio de Villena", 1987, pp. 157 y 88.

${ }^{19}$ AHN, Osuna, leg. 98, n. 3.

${ }^{20}$ Véase los trabajos sobre El Testainento de don Pedro Girón, y Morón y Osuna en la Baja Edad Media, ob. cit.
} 
xas, aunque en este caso concreto se inserten en una dinámica de guerra civil.

En síntesis podemos afirmar que la herencia que reciben los condes de Ureña de don Pedro Girón no sólu presenta aspectos positivos: creación de un linaje, vinculación en mayorazgo, etc. en definitiva la creación del Señorío de Osuna, sino que junto a ellos destaca una faceta más desconocida y que repercutió desfavorablemente en el propio Señorío.

Estos tres casos que hemos analizado son solamente una muestra de las cuantiosas deudas e indemnizaciones a las que los miembros de la nobleza deben hacer frente para sufragar los daños ocasionados por algún miembro de su familia. Si bien es verdad que las cantidades totales no suponen una ruina para el señorfo, si es necesario tenerlas en cuenta. En este caso concreto don Juan Téllez-Girón asumirá las de su padre en detrimento de las rentas del señorío de Osuna.

\section{RÉSUMMÉ}

Les testaments constituent unc intéressante source pour connaitre les attitudes et comportements individucls, comme on peut l'observer dans celui du Maître de Calatrava, Pedro Girón, mais surtout il nous informe sur les dettes et indemnités payées par ses heriticrs, en particulicr par le deuxième comte, Juan Téllcz. Girón. Celui-ci fut obligé d'acquitter les dettes de son père, que l'on peut répartir en trois categories: dettes particulières, comme les compensations à Juana González Almagro; dettes publiques liées à la creation du posito de Jaćn; et dettes relevant de l'ćxćcution testamentaire associée à la fondation d'unc chapellenic à Belmontc.

\section{SUMMARY}

The wills constitute an interesting source to know the attitudes and individual behaviours, as is observed upon analyzing the testament of the Master of Calatrava Don Pedro Girón, and above all the obligations and compensations that his heirs and very especially the Second Count of Ureña, Don Juan Téllez Girón, had to face. He had to pay the large debts contracted by his father, that we can classify at three levels: individual debts, such as the compensation to Juana González at Almagro; debts entailed to the foundation of the posito of Jaén; debts due to the execution of testamentary dispositions that ordered the foundation of a chaplaincy at Belmonte. 